\title{
EXAMPLE OF A SINGLE-VALUED FUNCTION \\ WITH A NATURAL BOUNDARY, WHOSE INVERSE IS ALSO SINGLE-VALUED.
}

\author{
BY PROFESSOR W. F. OSGOOD.
}

(Read before the American Mathematical Society at the Meeting of April 30, 1898.)

That functions exist which are analytic within the unit circle, have the unit circle as a natural boundary, and take on no value more than once, can be readily shown.

Let $T$ be a region of the $u+v i$-plane bounded by a single curve, the tangent of which turns continuously as the point of tangency trace out the curve; then there exists, even in this case, which is more general than the cases considered by Schwarz and Neumann, a Green's function belonging to $T$,* so that the interior of $T$ can be mapped conformally $\dagger$ on the interior of the unit circle. Furthermore, the boundary of $T$ will, even in this case, go over into the boundary of the circle in such a manner as to render the transformation of the region consisting of $T$ and its boundary on the region consisting of the circle and its boundary one-to-one and continuous.

Let the curve which bounds $T$ be represented by the equations

$$
u=\varphi(t), \quad v=\psi(t),
$$

where $\varphi, \psi$ denote continuous functions having the primitive period unity, so that, when $t$ increases from $t_{0}$ to $t_{0}+1$, the point $(u, v)$ describes the boundary once. The corresponding point $(x, y)$ will then describe the unit circle once and the angle $\theta=\tan ^{-1} \frac{y}{x}$ will be a single-valued, continuous function of $t ; t$, a single-valued, continuous function of $\theta$.

* Poincaré's solution of the boundary value problem is sufficiently general to cover this case. Cf. Poincaré : "Sur les équations aux dérivées partielles de la physique mathématique," Amer. Jour. of Math., vol. 12 (1890) ; Paraf's Thesis : "Sur le problème de Dirichlet et son extension au cas de l'équation linéaire générale du second ordre," Paris, 1892, and the Toulouse Annales, vol. 6. An account of these papers is given in Picard's Traité d'Analyse, vol. 2, ch. 4.

† Inaugural Dissertation, \& 21; Göttingen, 1851.

†Cf. Painlevé : "Sur la théorie de la représentation conforme," Comptes Rendus, vol. $112(1891)$, p. 653. This paper is based in part on his Thesis : "Sur les lignes singulières des fonctions analytiques," Paris, 1887, and the Toulouse Annales, vol. 2 (1888). Painlevé points out that even on the boundary angles are preserved. 
Let $\varphi(t)$ be chosen as an analytic, $\psi(t)$ as a non-analytic function of $t$. Then the function $u+v i$ will have the unit circle of the $x+y i$-plane as a natural boundary. For, otherwise, at some point $a+b i$ of the boundary of the unit circle, $u+v i$ would be analytic and thus $u$ and $v$ would each be analytic functions of $\theta$ near $a+b i$ along the arc. Thus $t$, being an analytic function of $u$, would have to be an analytic function of $\theta$ in this neighborhood, and hence $v$, being an analytic function of $\theta$, would be an analytic function of $t$.

The unit circle of the $x+y i$-plane is then a natural boundary for the function $u+v i$. The inverse function, $x+y i$, is single-valued and analytic throughout $T$, but cannot be continued beyond $T$. For, if it were analytic at any point on the boundary of $T$, its inverse, $u+v i$, would be analytic at, or near, the corresponding point on the unit circle of the $x+y i$-plane.

The existence proof just given is wholly satisfactory as regards motif ; and it is rigorous. Nevertheless, it may not be without interest to give a simple explicit example of a function of this kind. Such an example is afforded by the series

$$
f(z)=z+\frac{z^{a+2}}{(a+1)(a+2)}+\frac{z^{a^{2}+2}}{\left(a^{2}+1\right)\left(a^{2}+2\right)}+\cdots,
$$

where a denotes an integer greater than unity.

The function

$$
\varphi(z)=z^{a}+z^{a^{2}}+z^{a^{3}}+\cdots=\sum_{1}^{\infty} z^{a^{n}}
$$

where $a$ denotes an integer greater than unity, is analytic within the unit circle and has the circle as a natural boundary. Exceedingly simple proofs for this example have been given by Hadamard $*$ and by Stäckel. $\dagger$

The function $f(z)$, of which $\varphi(z)$ is the second derivative, will also have the unit circle as a natural boundary. Both the series defining $f(z)$ and the series representing its derivative :

$$
f^{\prime}(z)=1+\frac{z^{a+1}}{a+1}+\frac{z^{a^{2}+1}}{a^{2}+1}+\cdots=1+\sum_{1}^{\infty} \frac{z^{a^{n}+1}}{a^{n}+1}
$$

converge uniformly throughout the unit circle inclusive of

* "Essai sur l'étude des fonctions données par leur développement de Taylor," Liouville, 4th ser. vol. 8 (1892), p. 115. The proof is well presented in Harkness and Morley's Theory of Functions, 8104 .

† Crelle, vol. 113 (1892), p. 262. 
the boundary, and hence $f(z)$ and $f^{\prime}(z)$ are continuous throughout this domain. Furthermore, when $|z| \leqq 1$,

$$
\begin{gathered}
\left|f^{\prime}(z)\right| \geqq 1-\frac{1}{a+1}-\left(\frac{1}{a^{2}+1}+\frac{1}{a^{3}+1}+\cdots\right) \\
>1-\frac{1}{a+1}-\left(\frac{1}{a^{2}}+\frac{1}{a^{3}}+\cdots\right)=1-\frac{1}{a+1}-\frac{1}{a(a-1)}>0 .
\end{gathered}
$$

Hence the neighborhood of any point $z$ lying within the unit circle is transformed conformally on the neighborhood of the corresponding point $w$, and thus the image in the $w$ plane contains no branch-points. This is, of course, not enough to justify the conclusion that the inverse function $z(w)$ is single-valued; for the image in the $w$-plane might overlap itself. To show that this is not the case, it is sufficient to prove that, $(\rho, \varphi)$ denoting the polar coördinates of the point $(u, v)$ that corresponds to the point $\theta=$ $\tan ^{-1} \frac{y}{x}$ on the unit circle, (1) $\rho$ always remains positive; (2) $\varphi$ always increases when $\theta$ increases; and (3) $\varphi$ advances only by $2 \pi$ when $\theta$ increases by $2 \pi$. For then, to a given point $(\rho, \varphi)$ on the curve in the $w$-plane, there can correspond but one point $0 \leqq \theta<2 \pi$ on the unit circle, and all of the conditions of the theorem * are fulfilled which says : Let $f(z)$ be a function of $z$ single-valued and continuous throughout a simply connected region inclusive of the boundary; and let $f(z)$ be analytic throughout the interior of the region. Furthermore, let a value that $f(z)$ takes on in one point of the boundary never be assumed in a second point of the boundary. Then to the boundary curve in the z-plane will correspond a closed curve in the w-plane that does not cut itself, and the region of the w.plane thus bounded will, together with its boundary, correspond in a one-to-one manner and continuously to the region of the z-plane above mentioned, the transformation being conformal in the neighborhood of any pair of corresponding interior points.

It remains then only to give the proofs of (1), (2), and (3). The values of $u, v$ when $r=1$ are

where

$$
u=\cos \theta+C(\theta), \quad v=\sin \theta+S(\theta),
$$

$$
C(\theta)=\frac{\cos (\alpha+2) \theta}{(\alpha+1)(a+2)}+\frac{\cos \left(\alpha^{2}+2\right) \theta}{\left(a^{2}+1\right)\left(\alpha^{2}+2\right)}+\cdots
$$

* Picard: Traité d'Analyse, vol. 2, p. 280. 


$$
S(\theta)=\frac{\sin (a+2) \theta}{(a+1)(a+2)}+\frac{\sin \left(a^{2}+2\right) \theta}{\left(a^{2}+1\right)\left(a^{2}+2\right)}+\cdots
$$

The derivatives of $C(\theta), S(\theta)$ are

$$
\begin{gathered}
C^{\prime}(\theta)=-\left[\frac{\sin (a+2) \theta}{a+1}+\frac{\sin \left(a^{2}+2\right) \theta}{a^{2}+1}+\cdots\right] \\
S^{\prime}(\theta)=\frac{\cos (a+2) \theta}{a+1}+\frac{\cos \left(a^{2}+2\right) \theta}{a^{2}+1}+\cdots
\end{gathered}
$$

Since

$$
\begin{gathered}
\varphi=\tan ^{-1} \frac{v}{u}, \\
d \varphi=\frac{u d v-v d u}{u^{2}+v^{2}} .
\end{gathered}
$$

$$
u d v-v d u=\left[1+\left\{S^{\prime}(\theta) \cos \theta-C^{\prime}(\theta) \sin \theta\right\}\right.
$$

$\left.+\{C(\theta) \cos \theta+S(\theta) \sin \theta\}+\left\{C(\theta) S^{\prime}(\theta)-C^{\prime}(\theta) S(\theta)\right\}\right] d \theta$, $u^{2}+v^{2}=1+2\{C(\theta) \cos \theta+S(\theta) \sin \theta\}+C^{2}(\theta)+S^{2}(\theta)$.

A simple trigonometric reduction yields the formulas

$$
\begin{gathered}
S^{\prime}(\theta) \cos \theta-C^{\prime}(\theta) \sin \theta \\
=\frac{\cos (a+1) \theta}{a+1}+\frac{\cos \left(a^{2}+1\right) \theta}{a^{2}+1}+\cdots, \\
C(\theta) \cos \theta+S(\theta) \sin \theta \\
=\frac{\cos (a+1) \theta}{(a+1)(a+2)}+\frac{\cos \left(a^{2}+1\right) \theta}{\left(a^{2}+1\right)}+\cdots .
\end{gathered}
$$

Now $C(\theta), S(\theta), C(\theta) \cos \theta+S(\theta) \sin \theta$ do not exceed numerically the value of the series

$$
\begin{gathered}
\frac{1}{(a+1)(a+2)}+\frac{1}{\left(a^{2}+1\right)\left(a^{2}+2\right)}+\cdots \\
<\frac{1}{a \cdot a}+\frac{1}{a^{2} \cdot a^{2}}+\cdots<\frac{1}{a^{2}-1}
\end{gathered}
$$

and $C^{\prime}(\theta), S^{\prime}(\theta), S^{\prime}(\theta) \cos \theta-C^{\prime}(\theta) \sin \theta$ do not exceed numerically the value of the series

$$
\frac{1}{a+1}+\frac{1}{a^{2}+1}+\cdot \cdot<\frac{1}{a}+\frac{1}{a^{2}}+\cdots<\frac{1}{a-1}
$$


Proof of (1)

$$
\begin{aligned}
& \left|2\{C(\theta) \cos \theta+S(\theta) \sin \theta\}+C^{2}(\theta)+S^{2}(\theta)\right| \\
& <\quad 2 \frac{1}{a^{2}-1}+\frac{1}{\left(a^{2}-1\right)^{2}}+\frac{1}{\left(a^{2}-1\right)^{2}}
\end{aligned}
$$

The largest value this expression can have is that corresponding to $a=2, i$. e., $\frac{8}{9}$, and thus (1) is established.

Proof of (2), (3). Similarly the coëfficient of $d \theta$ cannot become negative if $a>2$, for it is surely greater than

$$
1-\frac{1}{a-1}-\frac{1}{a^{2}-1}-2 \frac{1}{(a-1)\left(a^{2}-1\right)},
$$

i. e., than $\frac{1}{4}$. For such a value of $a$,

$$
d \varphi<\frac{1+\frac{1}{a-1}+\frac{1}{a^{2}-1}+2 \frac{1}{(a-1)\left(a^{2}-1\right)}}{1-2 \frac{1}{a^{2}-1}} d \prime^{\prime}
$$

and if $a>3, d \varphi<2 d \theta$, so that $\varphi_{\theta=2 \pi}<4 \pi$.

The proof is now complete for all values of $a$ greater than 3. For these values of $a$ the requisite analysis was very simple. For the values $a=2$ and $a=3$, however, the above approximations are not close exough for (2) and (3). For these cases (3) may be established as follows:

$$
\begin{array}{ll}
\text { where } & \Phi(\theta)=\left[u^{2}+v^{2}\right]^{-1} \\
\text { and } & F(\theta)=v \frac{d u}{d \theta}-u \frac{d v}{d \theta}
\end{array}
$$

By a well known law of the mean

But

$$
\varphi_{\theta=2 \pi}=\Phi\left(\theta_{1}\right) \int_{0}^{2 \pi} F(\theta) d \theta, \quad 0<\theta_{1}<2 \pi
$$

$$
\Phi\left(\theta_{1}\right)<\frac{1}{1-2 \times .133}<\frac{10}{7},
$$

as appears from the closer approximation for

$$
|C(\theta) \cos \theta+S(\theta) \sin \theta|
$$

given on the next page, and

$$
\begin{gathered}
\int_{0}^{2 \pi} F(\theta) d \theta=2 \pi+0+0+\lambda, \\
\text { where } \lambda=\int_{0}^{2 \pi}\left\{C(\theta) S^{\prime}(\theta)-C^{\prime}(t) S(\theta)\right\} d \theta
\end{gathered}
$$




$$
\begin{gathered}
<\int_{0}^{2 \pi} 2\left[\frac{1}{a+1}+\frac{1}{a(a-1)}\right]\left[\frac{1}{(a+1)(a+2)}\right. \\
\left.+\frac{1}{a^{2}\left(a^{2}-1\right)}\right] d \theta \leqq \frac{5}{18} \cdot 2 \pi
\end{gathered}
$$

Hence

$$
\varphi_{\theta=2 \pi}<\frac{10}{7}\left[1+\frac{5}{18}\right] 2 \pi<4 \pi
$$

It remains then only to give the proof of (2) when $a=2$. This can be done as follows:

$$
\begin{gathered}
\left|S^{\prime}(\theta) \cos \theta-C^{\prime}(\theta) \sin \theta\right|<\frac{1}{3}+\frac{1}{5}+\frac{1}{9}+\frac{1}{17}+\frac{1}{33} \\
+\frac{1}{2^{5}(2-1)}<.765 \\
|C(\theta) \cos \theta+S(\theta) \sin \theta|<\frac{1}{12}+\frac{1}{30}+\frac{1}{90} \\
+\frac{1}{2^{6}\left(2^{2}-1\right)}<.133
\end{gathered}
$$

To get a sufficiently close approximation for the remaining parenthesis, multiply out the series for $C(\theta)$ and $S^{\prime}(\theta)$; let the result be written as

$$
\sum_{n=1}^{\infty} \alpha_{n} \cos p_{n} \theta \cos q_{n} \theta
$$

where $p_{n}, q_{n}$ are positive integers or zero. Now the series $-C^{\prime}(\theta)$ and $S(\theta)$ when multiplied out give

$$
\sum_{n=1}^{\infty} \alpha_{n} \sin p_{n} \theta \sin q_{n} \theta
$$

Hence it follows that

$$
\left|C(\theta) S^{\prime}(\theta)-C(\theta) S(\theta)\right|<\sum_{n=1}^{\infty} \alpha_{n}<.765 \times .133
$$

and since $.765+.133+.765 \times .133<1$ the proof is complete.

I have thought it worth while to call attention to the existence of functions of the kind have treated, because they are useful in theoretical investigations. To cite a single instance: The theorem that an analytic function of an analytic function is an analytic function is true im Kleinen, but not necessarily im Grossen. The nature of the restrictions that are necessary for the truth of the theorem is readily demonstrated $a d$ oculos by the aid of the above ex- 
ample. Begin by drawing in the $z$-plane a circle of unit radius with its centre in the point $z=1+i$, and let $\Phi(z)$ be any function analytic within this circle, but not capable of analytic extension beyond it - for example

$$
\Phi(z)=f(z-1-i) .
$$

Next, let $z^{3}=z^{\prime}$. The circle is then transformed conformally on a region not including the origin, lying wholly within the first, second and third quadrants, and tangent to the axis of reals in the point $z^{\prime}=1$, to the axis of imaginaries in the point $z^{\prime}=-i$. Let

$$
\Phi(z)=F\left(z^{\prime}\right) .
$$

Then $F(z)$ (the accent being dropped) is single-valued and cannot be continued analytically beyond this region. Now
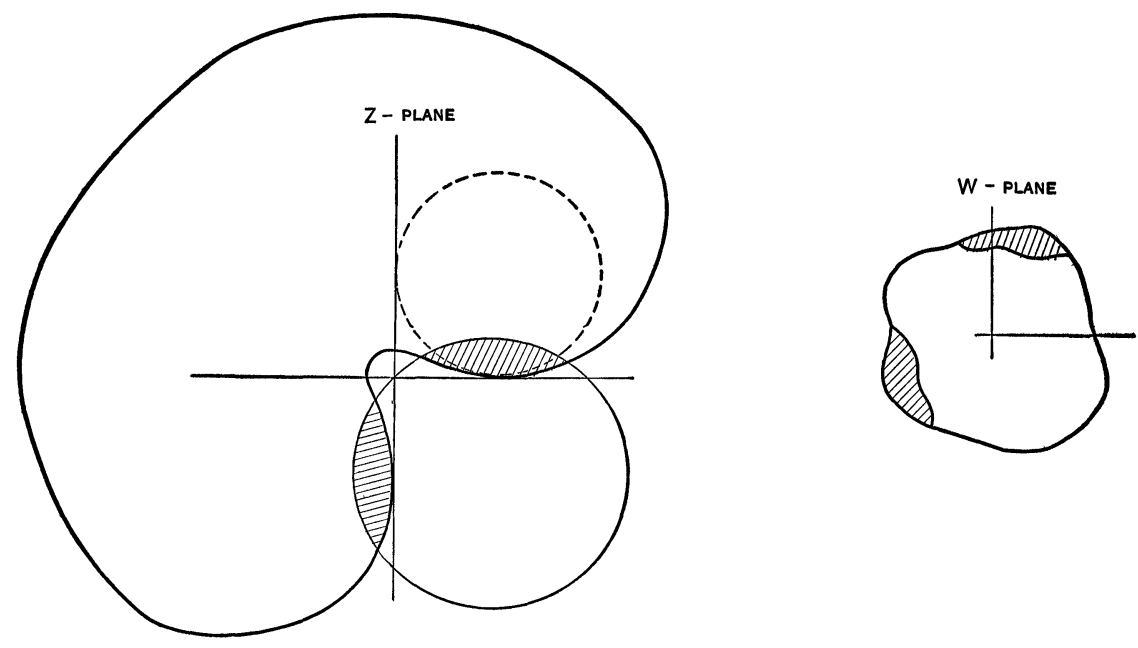

draw a circle passing through the point $z=0$ and including the points $z=1, z=-i$ in its interior - for definiteness, let its centre be at the point $z=1-i$; its radius will then be equal to $\sqrt{2}$. The function

$$
w=f\left[\frac{z-1+i}{\sqrt{2}}\right]
$$

will map this circle conformally on the region of the $w$-plane above considered, and $z$ will be a single-valued analytic function of $w$. 
Consider those values of $w$ that yield values of $z$ for which $F(z)$ is defined, and for which then $F(z)$ is a function of $w$. These values of $F(z)$ do not constitute an analytic function of $w$; for the domain of values of $w$ consists of two separate continua. Thus the theorem, unrestricted, would be false in this case. *

HARVARD UNIVERSITY, April, 1898.

\title{
NOTE ON POISSON'S INTEGRAL.
}

\author{
BY PROFESSOR MAXIME BÔCHER.
}

(Read before the American Mathematical Society at the Meeting of April $30,1898$.

THe following treatment of Poisson's integral in two dimensions seems to the writer to have at least one advantage over the treatments ordinarily given; viz., that it involves no artifice.

Given a function $V(x, y)$ which within and upon the circumference of a certain circle $C$ is a continuous function of $(x, y)$ and within $C$ is harmonic $(i$. e., has continuous first and second derivatives and satisfies Laplace's equation). By a well-known theorem of Gauss the value of $V$ at the centre $\left(x_{0}, y_{0}\right)$ of $C$ is the arithmetic mean of its values on the circumference. $\dagger$ That is, if we denote by $V_{c}$ the values of $V$ on the circumference and by $\varphi$ the angle at the centre,

$$
V\left(x_{0}, y_{0}\right)=\frac{1}{2 \pi} \int_{0}^{2 \pi} V_{c} d \varphi \text {. }
$$

This theorem may be immediately generalized by the method of inversion, if we remember on the one hand that a harmonic function remains harmonic after inversion, and on the other hand that angles are unchanged by inversion and that circles invert into circles. We thus get the theorem :

* Burkhardt has given simple examples of multiple-valued functions for which the unrestricted theorem is false. See his book : "Einführung in die Theorie der analytischen Functionen einer complexen Veränderlichen," vol. 1, Leipzig, 1897 ; p. 198.

$\dagger$ An elementary proof of this theorem will be found in a paper by the writer on p. 206 of the BuLLETIN for May, 1895. 